\title{
The upgrade in human evolution? It's a matter of TIME!
}

Professor Sahra Talamo

Alma Mater Studiorum, Bologna University, Italy

We are all fascinated with time. We always want to organise it, decide it, define it, and improve it as a means of improving ourselves. Knowing the time makes us understand our reality, feel alive, feel present, and guess what? We are the only human species present here today! We are the result of a finely tuned evolution. Not only have we evolved, but we are constantly upgrading, "WHATEVER IT TAKES!".

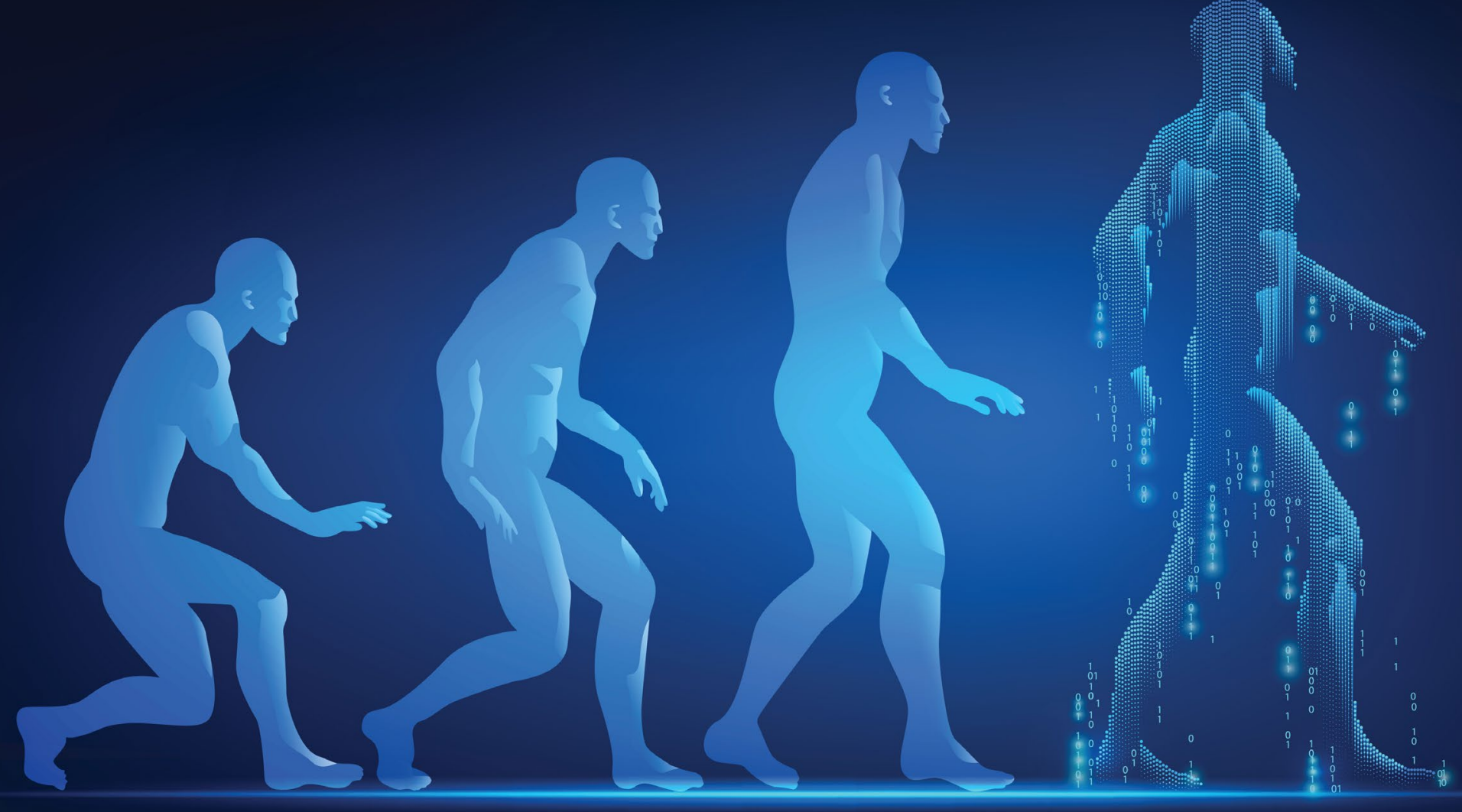

As the Dalai Lama said, "if we want to improve the world, we must first improve ourselves". As an archaeologist who it is hard to improve ourselves if we do not know our past and our mistakes. let me be imperfect in using the pest Let me be imperfect in aring the past perfect to emphasize a 40,000 years of past, a glonious past the brought us her who 'we had been'?

In the fascinating story of human evolution, our awareness of time that 'had been' is marked by the atomic clock, the radiocarbon ( $\mathrm{C}$ ). Understanding how it works and how it can be improved in studying human evolution is essenti if we want to UPGRADE.

What do we not yet know about human evolution?

Around 40,000 years ago, Europe was at in heart of the most faschnating events in human evolution. This period saw the unfolding of the dramatic events between Neanderthals, our closest cousins, and us, Homo sapiens. What exactly happened when Homo sapiens arrived from Africa and met the European Neanderthals still veiled in mystery and has stimulated research for more than a century.

We all are fascinated by the new advances in palaeogenetic studies proving that Neanderthals contributed to the genetic varlation of present-day humans by mixing with Homo sapiens when they spread in Eurasia. Nowadays, the genome of Europeans is about 2 per cent closely related to the Neanderth genome, and some of the medica conditions affecting our skin, blood and the risk of depression are considered due to this archaic genomic admixture.

Neanderthals lived in Europe for more than 300 thousand years with slow changes in culture and technolosy. Only at the end of their history did new technical behaviour emerse, just a few millennia before their demise. These new techno-complexes, named 'transition (e.g. the Châtelperronian in France and northern Spain the Szeletian in the Czech Republic and Hungary), are the topic of intense debates. It is still unknown these behavioural changes result fro Neanderthals endemic innovations o cultural exchanges with Homo sapiens during their fist waves of expansion into these new territories. Solving th teritor solve the Evolution. To understand who 'we h ' been' and the success that brought us such How long did we share the territory? Did we influence their culture? Why did they disappear, and we survived?

In this panorama, chronology plays a pivotal role, with the radiocarb 'clock' representing the backbone chronological reconstructions for the time up to 55,000 years ago (55 ka BP, Before Present).

What is radiocarbon dating?

Since Libby won the Nobel Prize in Chemistry in 1960, radiocarbon dating has been the most widely used dating method in the field of archaeology. Personally, I think that radiocarbon is a 'gift from heaven' because it is form in the upper atmosphere, incorporated into all living organisms, and decays over time. " $\mathrm{C}$ has a beta decay of 5,730 years; this low rate of decay is unique, allowing us to measure the amount of radiocarbon left in an organic sample and thus determine the time of death, $u p$ to 55,000 years. Its only flaw is that the amount of radiocarbon in the atmosphere varied over time. Therefore, the radiocarbon clock must be calibrated to an independent calendar scale to obta calendar age of archaeological samples. This is what we call the calibration curve.

For the past 14,000 years, the calibration curve has been based on trees, and it is highly precise. For older periods, where trees were not measured, the precision of the calibration curve drops dramatically. The further we travel into the past, the less accurate radiocarbon dates become, with substantial errors of 1,500 years and more.

This situation leaves scientists navigating in a sea of imprecision, especially when discussing the interaction between Neanderthals and Homo sapiens. In a historical context, this uncertainty would be equivit to saying the be equivat the Curie sits alongside Archinedes at the Solvay congress instead of sitting between Max Planck and Einstein.

How can the problem of accuracy in radiocarbon dating be resolved?

The radiocarbon clock has always ticked with two hands. To increase the accuracy of the time intervals that mark the course of our history, we need to add a third hand, and we need to combine different scientific disciplines and different points of view to do that. We should have a multidisciplinary approach, because without the multidisciplinarity, there would be no solutions to complex problems. Our field of human evolution interacting with radiocarbon poses problems that would be difficult to solve if we wanted to solve them alone. This is where the RESOLUTION project comes in. 


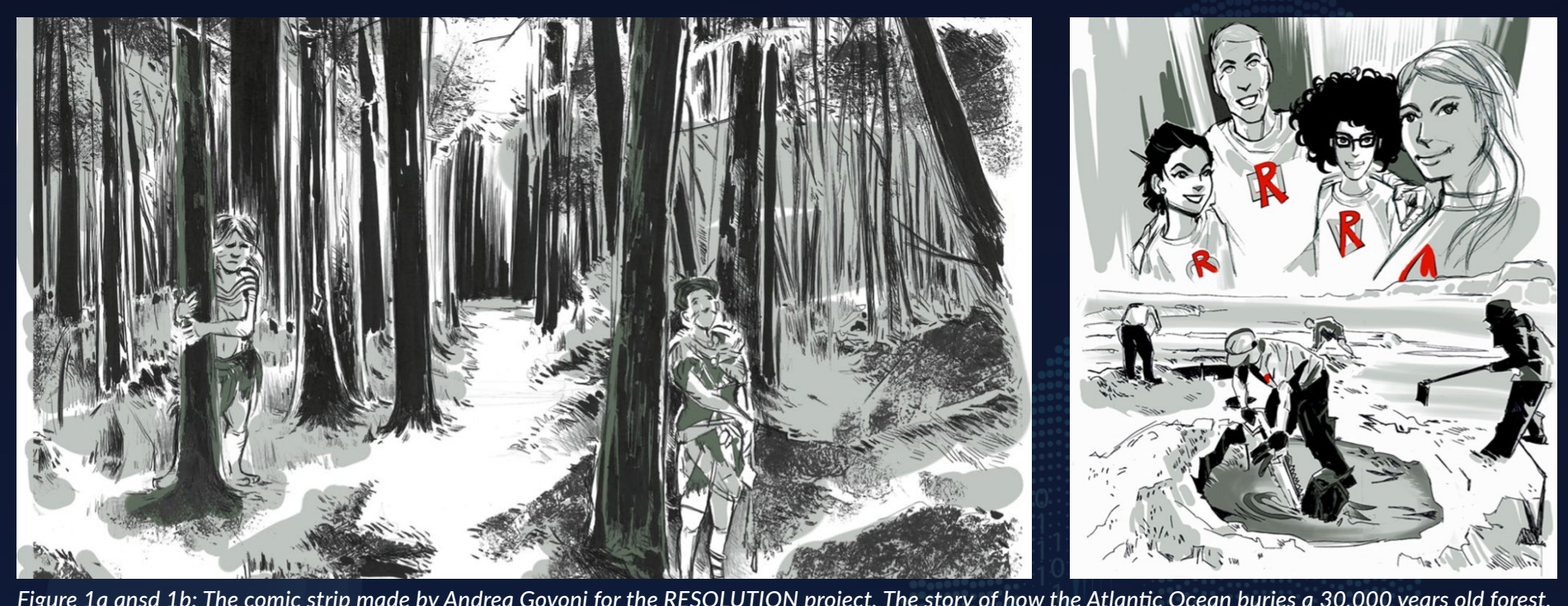

Figure 1a ansd 1b: The comic strip made by Andrea Govoni for the RESOLUTION project. The story
and the RESOLUTION team brings it back to life. The illustrations are under RESOLUTION copyrigh

The first step is to research and study fossil trees, which make the first part of the curve so accurate but wereco not to be present before 14,000 years. There have been fossil forests in Europe during glacial times; you just have to look for them. We found one of 30,000 years old right under the Atlantic Ocean on the shores of Portugal (Figures 1a, 1b and 3 .

The second step is to incorporate a brand new method from physics, which exploits the synchronicity of the cosmogenic isotopes of ${ }^{14} \mathrm{C}$ and Beryllium-10 $\left({ }^{10} \mathrm{Be}\right.$ We can join the ${ }^{14} \mathrm{C}$ dates obtained from glacial trees to the calendar age obtaine from the annual presence of ${ }^{10} \mathrm{Be}$ in the layers of Greenland ice cores. In this way we can start building new curve pieces that will give us very precise ages (Figure 2). However, be careful; this method need very high precision, a very small ${ }^{14} \mathrm{C}$ error, fewer than 300 years. This is not eas Typically, from the analysis of a sample of 42,000 years, routine analysis gave errors of around 1,700 years. This is why Marie Curie sits at the side of Archimedes.

Thanks to the collaboration of physicists working with the most advanced dating machine (AMS-MICADAS), we have been able to obtain for this time period $(42,000)$ before $(42,271 \pm 296$ BP) (Hublin et al. 2020; Fewlass et al. 2020). This will allow us to study prehistoric climate

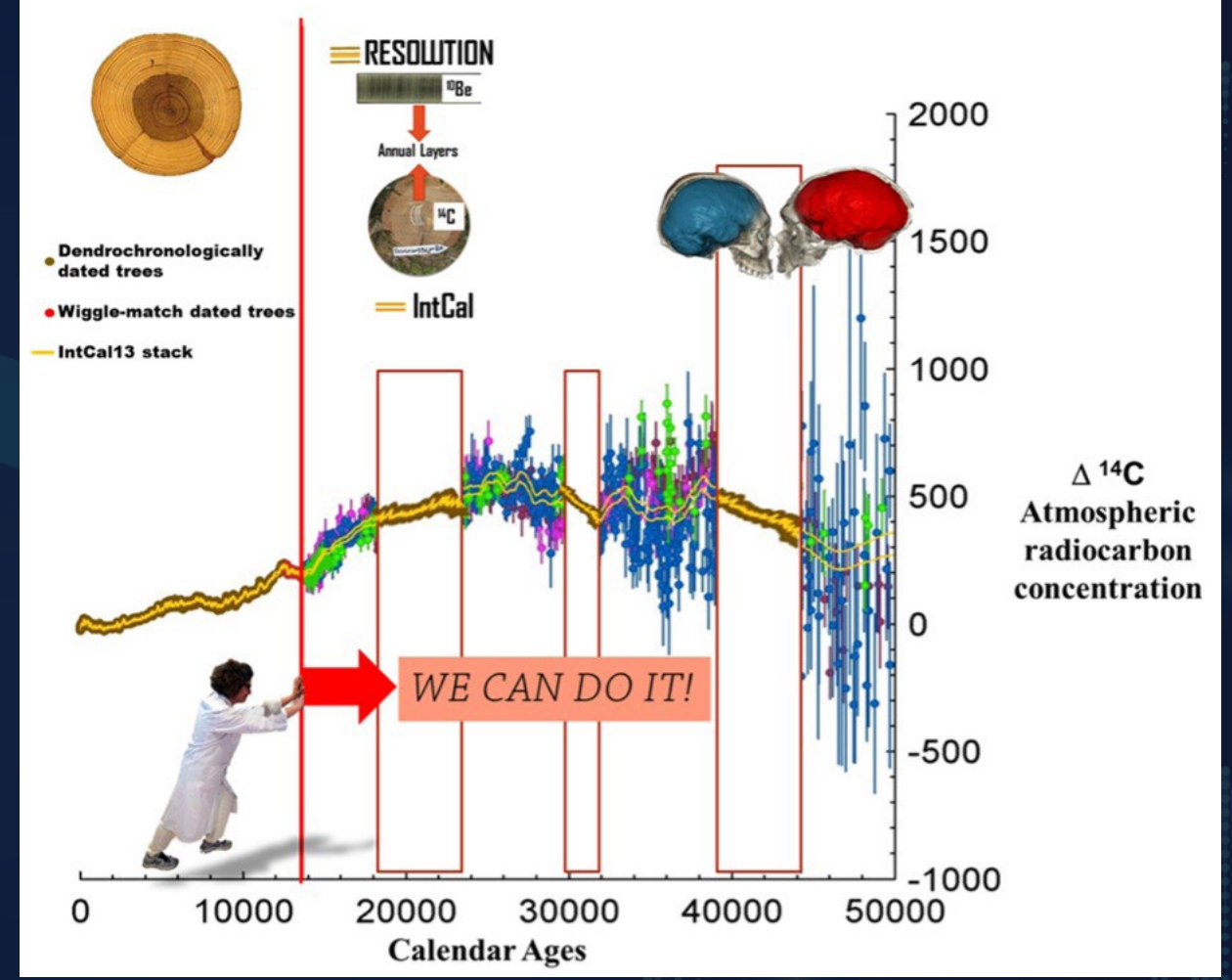

Figure 2: Example to show the goal of the RESOLUTION project. and provide outstanding resolution for European Prehistory.

What challenges have the RESOLUTION team faced during the COVID-19 pandemic?

Doing research is never easy, especially if you have to move from an institute dedicated solely and exclusively to research, such

In my case, Alma Mater Studiorum, "The mother that feeds studies" and "The University is where dediction to the students have pro and dedication to the students have priority. However, the love for the research is in this is the

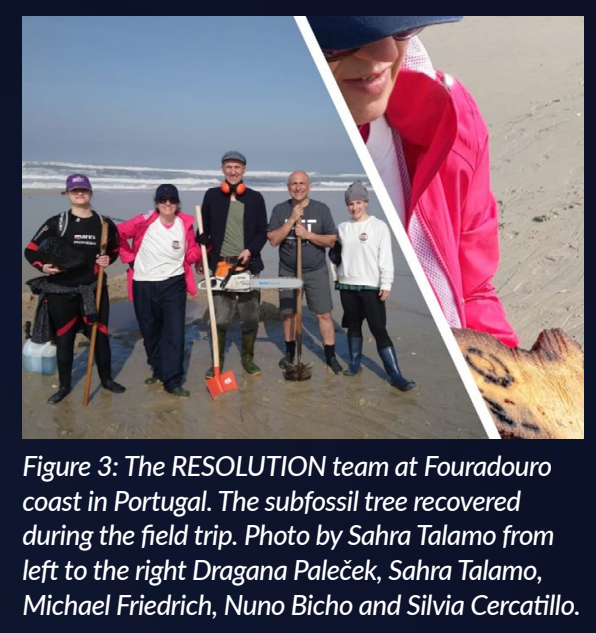

Creating a research group with students that have passion and desie students is not always easy, and I must say that is not always easy, and I must say that from this point of view, I have been very

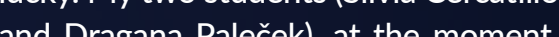
and Dragana Pallek), at the moment, are not only full of passion, but they are keen to learn and try to catch the sightest positive sign of research. With this spirit, we left Bologna in a van together with my trusty PostDoc in Dendrochronology from Hohehnehim University, Germany (Dr Michae Friedrich). The direction was Portugal search of our first fossil trees.

Leaving Italy on 7 March 2020, during an 'apparently' ordinary day since COVID was almost at the gate, we managed to arrive after several stops (Switzerland, France, Spain) in Portugal. At Furadouro, under the Atlantic Ocean, under the sand still wet from the sea in a rare day of low tide, stood a fossil forest of 30,000 years ago (Figure 3). Amazingly, we managed to find a few of the trees still in a living position before the high tide returned to bury them once again until the next low tide, months away.

As we splashed away from Portugal, behind us, the gates of all the cities regions were closing and the 'red zone' regions were closing and the 'red zone countries just in time to be locked in for a whole year.

What to do? Luckily we had the first trees with us. However, the original working plan could not be implemented. What to do? How to move forward?
Well, the mind of a researcher never sleeps, never stops focusing on the goal you want to achieve, not on Saturdays Sundays, not at Christmas, and certainly not during the COVID!

An Italian proverb says: "when the play gets tough, the player starts to play" and here we are.

I changed the programme. First, we started to put together the BRAVHO radiocarbon lab. Then we moved on to mmediately carry out the pretreatmen of wood samples in the lab and finis with the first methodological artic Cercatillo et al. 2021). We acquire new laboratory technician (Lau Tassoni) and many innovative machines for ${ }^{14} \mathrm{C}$ dating. We dedicated ourselve to the writing of as many scientific articles as possible.

Obviously, our initial dreams are temporarily on hold. But, if everythin goes as planned, we should resume our travels in October 2021

What's next for the RESOLUTION project?

Fieldwork, fieldwork and more fieldwork in several regions of the Mediterranean

My team and I will continue to: - fill gaps in knowledge on the presence of fossil trees in Europe in the glacial - refine the calibration curve using ou new segments

- provide exceptional radiocarbon precision for periods older the 15,000 years

- pinpoint exactly events in human

- clarify the role of climate in this story.

I strongly believe that with RESOLUTION, we will obtain new data and revisit existing information, to create what all of us right now can only dream of: a more precise and detailed timeline of our own story, the story of human evolution.

$\mathbf{R}$

\section{RESOLUTION RESOLUTEON}

PROJECT SUMMARY

RESOLUTION aims to achieve an accurate and highly resolved chronology back to 55,000 years BP interaction with Neanderthals and the final cause of Neanderthal extinction.

With tree-rings, the resolution will be an order of magnitude higher, and using the most recent advances in the AMS technique, we will obtain confidence intervals of only a few centuries in glacial times.

The project involves fieldwork in the Mediterranean and Southeast Europe to find more glacial trees, studying the existing collection of glacial conifers, exceptional ${ }^{14} \mathrm{C}$ Drecision for ${ }^{12} \mathrm{C}$ dates in the glacial, and the (ree-ring che methology linking floating tree-ring
scale.

PROJECT LEAD

Professor Sahra Talamo has been in the Department of Chemistry G. CIAMICIAN project lead for RESOLUTION. Talamo is the author of several international scientific papers concerning the chronology and interaction
between the Neanderthal and Homo sapiens. between the Neanderthal and Homo sapiens.
One of her recent papers, published in the Nature journal, is the 'Radiocarbon dating of the oldest European Homo sapiens'.

\section{CONTACT DETAILS}

Professor Sahra Talamo

(BRAVHO: Bologna Radiocaton

devoted to Human Evolution)

Department of Chemistry G Ciamicin

Alma Mater Studiorum, Bologna University

Via Selmi 2, I-40126 Bologna, Italy

푱 +390512099476

\sahra.talamo@unibo.it

www.unibo.it/sitoweb/sahra.talamo

@ @erc_resolution

/erc_resolution

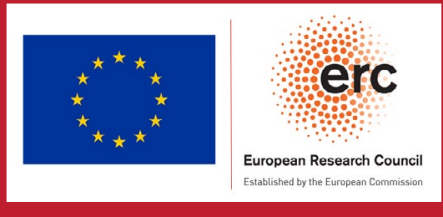

FUNDINC

This project has received funding from the European
Research Council (ERC) under the European Union's Horizon 2020 research and innovation programme under grant agreement №.803147. 


\section{DISSEMINATION RESOLUTION References}

\section{The upgrade in human evolution? It's a matter of TIME!}

Hublin, J.J., Sirakov, N., Aldeias, V., Bailey, S., Bard, E., Delvigne, V., Endarova, E., Fagault, Y., Fewlass, H., Hajdinjak, M., Kromer, B., Krumov, I., Marreiros, J., Martisius, N. L., Paskulin, L., Sinet-Mathiot, V., Meyer, M., Pääbo, S., Popov, V., Rezek, Z., Sirakova, S., Skinner, M. M., Smith, G. M., Spasov, R., Talamo, S., Tuna, T., Wacker, L., Welker, F., Wilcke, A., Zahariev, N., Mcpherron, S. P. and Tsanova, T. (2020) 'Initial Upper Palaeolithic Homo sapiens from Bacho Kiro Cave', Bulgaria. Nature, 581, pp.299-302.

Fewlass, H., Talamo, S., Wacker, L., Kromer, B., Tuna, T., Fagault, Y., Bard, E., Mcpherron, S. P., Aldeias, V., Maria, R., Martisius, N. L., Paskulin, L., Rezek, Z., Sinet-Mathiot, V., Sirakova, S., Smith, G. M., Spasov, R., Welker, F., Sirakov, N., Tsanova and T., Hublin, J.J. (2020) 'A 14C chronology for the Middle to Upper Palaeolithic transition at Bacho Kiro Cave, Bulgaria', Nature Ecology \& Evolution, 4, pp.794-801.

Cercatillo, S., Friedrich, M., Kromer, B., Paleček, D. and Talamo, S. (2021) 'Exploring different methods of cellulose extraction for 14 C dating', New Journal of Chemistry, 45(20), pp.8936-8941. 